\title{
The Diversification of Proto-Cells Driven by Membrane Permselectivity
}

\author{
Masaomi Hatakeyama *† \\ School of Knowledge Science \\ Japan Advanced Institute of Science and Technology \\ 1-1, Asahidai, Nomi, Ishikawa, Japan, 923-1292 \\ masaomi.hatakeyama@gmail.com \\ http://homepage3.nifty.com/masaomi/ \\ Takashi Hashimoto \\ School of Knowledge Science \\ Japan Advanced Institute of Science and Technology \\ 1-1, Asahidai, Nomi, Ishikawa, Japan, 923-1292 \\ hash@jaist.ac.jp \\ http://www.jaist.ac.jp/ hash/index-e.html
}

*corresponding author

†Present address: TOYOTA CENTRAL R\&D LABS., INC. 41-1, Aza Yokomichi, Oaza Nagakute, Nagakute-cho, Aichi-gun, Aichi-ken, 480-1192, Japan 


\begin{abstract}
We consider how permselectivity as a function of the membrane is related to the cell evolution with an abstract proto-cell model. We construct an autopoietic proto-cell model having primitive auto-catalytic reaction cycle inside. In this model, several primitive membrane substances are assumed to be produced from the reaction cycle and the membrane is assumed to have a permeability to specific chemical species. We show that the permselectivity of the membrane induces the diversification of the cell volume. We discuss how the diversification of the cells is related to the Darwinian evolution.
\end{abstract}

Keyword : Cell evolution, Proto-cell model, Membrane permselectivity, Auto-catalytic reaction, Diversification. 


\section{Introduction}

Recently there has been taken into account the role of membrane in the prebiotic evolution. In particular, Ségre proposed 'Lipid World hypothesis' in 2001[9] as alternate of 'RNA World hypothesis'[4]. The Lipid World hypothesis is related to autocatalytic type of cell models already proposed by Varela and Maturana's Autopoiesys System[12], Gánti's Chemoton[3], and Oparin's Coaservate[8] etc. In this hypothesis not one molecule but an aggregate of amphiphilic molecules can reproduce itself without molecules carrying genetic information and can evolve on the prebiotic earth. In fact, the amphiphilic molecules are spontaneously self-assembled in aqueous solution and compose a spherical vesicle. There have been also some experiments recently to succeed the self-reproduction of lipid vesicles[1, 11]. It should be, however, considered what will happen after the reaction which can produce membrane substances is separated from the environment. It is particularly important to make sure whether the Darwinian evolution of the reaction cycle can occur or not and to elucidate the relation between the Darwinian evolution and the membrane functions.

The following three conditions are necessary for the Darwinian evolution[2].

1. Variation: There are differences among individuals.

2. Inheritance: The differences can be taken over to the descendant.

3. Selection: The differences influence the reproduction rate and the survival rate of the individuals. 
The livings which fit an environment remain if these conditions above are met.

In the prebiotic condition, a proto-cell seems to be able to satisfy the conditions for inheritance and selection. If a proto-cell is divided into two ones, the properties of the proto-cell can be inherited into the daughter proto-cells, and the proto-cells which have higher reproduction rate can be selected. It is, however, difficult to consider how the variation of proto-cells is generated and maintained without genetic molecules in the prebiotic condition.

We claim that the variation of proto-cells can be generated and maintained by one of membrane functions. The membrane permselectivity which allows certain substances to permeate the proto-cell membrane can construct a specific internal condition and this effect may cause the variation of the reproduction and survival rate.

The purpose of this study is to answer a question: How does the membrane permselectivity make the diversification of proto-cell possible? For this purpose we construct not a realistic and complicated cell model but a simple and conceptual model. Although a simple model is sometimes not compared quantitatively with any real objects, it is often useful to propose a new concept or a fundamental feature of complex systems in which some elements mutually interact with each other. Because of less parameters of the model it is easier and faster to analyze the interaction among elements and to find a fundamental mechanism of the system than a realistic one. In this sense, it can be often helpful for abductive inference and for making a working hypothesis of experimental research in the real world. We call this sort of scientific methodology 
'Constructive Approach'[7, 6].

\section{Proto-Cell Model}

We consider a simple proto-cell model which satisfies the following conditions.

1. It has an auto-catalytic reaction system inside.

2. Membrane substances are produced from the auto-catalytic reaction.

3. Membrane substances are self-assembled into a semipermeable membrane which separates the internal auto-catalytic reaction from the external environment.

The image of proto-cell model is shown in Figure 1. In the reaction $k$ kinds of chemical species, $\mathrm{X}_{1}, \ldots, \mathrm{X}_{k}$, react auto-catalytically and some membrane substances, $\mathrm{M}_{i}$, are produced from some of chemical species in the auto-catalytic reaction cycle as follows.

$$
\begin{aligned}
\mathrm{X}_{i}+\mathrm{X}_{i+1} & \rightarrow 2 \mathrm{X}_{i+1}\left(i=1, \ldots, k ; \mathrm{X}_{k+1} \equiv \mathrm{X}_{1}\right) \\
\mathrm{M}_{j} & \leftrightarrow \mathrm{X}_{j} \quad(j \in\{1, \ldots, k\})
\end{aligned}
$$

In Figure 1, the number of reaction chemical species is $k=4$ and memrane substances are produced from $\mathrm{X}_{3}$ and $\mathrm{X}_{4}(j=3,4)$.

We assume the following conditions:

- Inside of the cell is well-mixed and the concentration of each chemical is homogeneous in the cell. 
- The volume of the cell, $V$, is proportional to the amount of membrane substances.

- The molecules permeate and diffuse through the membrane with the diffusion coefficient $D_{i}$ for the $i$ th chemical species.

We use a stochastic method for calculating all the reaction processes and consider the number of molecules discretely. The effects of the discreteness of molecules and the smallness of the number of molecules should be considered in the actual cell[10].

We use Gillespie's Direct Method[5]. The Probability of each reaction is calculated as the following equation,

$$
P_{R i}=r_{i} x_{i} x_{i+1} V=\frac{r_{i} N_{i} N_{i+1}}{V}
$$

where $r_{i}$ is the reaction rate constant, $x_{i}$ is the concentration of chemical species $\mathrm{X}_{i}, V$ is the volume of the cell, and $N_{i}$ is the number of chemical species. The reaction probability of producing membrane substances is calculated from the Equation 4.

$$
P_{C_{i}}=c_{i} x_{i} V=c_{i} N_{i}
$$

where $c_{i}$ is the reaction rate constant of producing membrane substances. And the probability of reverse reaction is calculated from Equation 5.

$$
P_{B_{i}}=b_{i} m_{i} V=b_{i} Z_{i}
$$

where $b_{i}$ is the rate constant of the reverse reaction, $m_{i}$ and $Z_{i}$ are the concentration and the number of membrane substances, $\mathrm{M}_{i}$, respectively. The probability 
of efflux of chemical species from inside of the cell into the external environment is calculated from Equation 6 .

$$
P_{O i}=D_{i} x_{i} V=D_{i} N_{i}
$$

where $D_{i}$ is the diffusion coefficient of efflux of chemical species, $\mathrm{X}_{i}$. On the other hand, the probability of influx of chemical species is calculated from

$$
P_{I i}=D_{i} s_{i} V
$$

where $s_{i}$ is the concentration of the $i$ th chemical species in the external environment.

\section{$3 \quad$ Results}

The parameters in all the simulations are set up as follows:

- The number of chemical species $k=4$.

- All the reaction rate constants $r_{i}=0.1 \quad(i=1, \ldots, 4)$.

- Membrane substances are only produced from $\mathrm{X}_{3}$ and $\mathrm{X}_{4}$. Namely, there are two membrane substances, $\mathrm{M}_{3}$ and $\mathrm{M}_{4}$.

- The reaction rate constant of membrane producing and the reverse reaction rate constant are $c_{j}=b_{j}=0.01 \quad(j=3,4)$.

- Every initial number of molecules is $N_{1}=N_{2}=N_{3}=N_{4}=Z_{3}=Z_{4}=$ 100. 


\subsection{Basic properties}

Figure 2(a)(b) show examples of the typical phenomena of each molecule without permselectivity in this model. Figure 2(a) shows the time-series of the number of chemical species when the diffusion coefficient $D_{i}=0.001 \quad(i=$ $1, \ldots, 4)$ for each molecule, and Figure 2(b) when $D_{i}=0.0001 \quad(i=1, \ldots, 4)$. In the case of higher diffusion coefficient, the number of molecules oscillates. On the other hand, when the diffusion coefficient is lower, only $\mathrm{X}_{1}$ and $\mathrm{X}_{3}$ oscillate and $\mathrm{X}_{2}$ and $\mathrm{X}_{4}$ are mostly zero after Time $=20000$. The opposite case, namely $\mathrm{X}_{2}$ and $\mathrm{X}_{4}$ oscillate and $\mathrm{X}_{1}$ and $\mathrm{X}_{3}$ are mostly zero, can occur with the same probability. The cell volume is almost constant all through the time without permselectivity irrespective of the diffusion coefficient.

\subsection{Diversification driven by permselectivity}

When each chemical species has a different permeability, the volume changes as a function of time. The time-series of cell volume are exemplified in Figure 3(a). In this figure 'Permselect: $p_{1} p_{2} p_{3} p_{4}$ ' designates the permselectivity pattern of membrane for each chemical species. $p_{i}=1$ means high permeability $D_{i}=0.001$, and $p_{i}=0$ means low permeability $D_{i}=0.0001$. For example, 'Permselect:1000' means $D_{1}=0.001, D_{2}=D_{3}=D_{4}=0.0001$. As shown in the previous subsection, in the case of no permselectivity, 'Permselect:0000' and 'Permselect:1111', the cell volume is almost constant all through the time. The former case is shown by the thin solid line in Figure 3(a). The cell volume is increasing as shown by the thin dashed line in Figure 3(a) in the case that only 
$D_{1}$ and $D_{2}$ are high value. The cell volume changes with big fluctuation when $D_{1}, D_{2}$, and $D_{3}$ are high (thick solid line in Figure 3(a)). The low value of only $D_{1}$ makes the cell volume tend to decay and terminate the reaction finally. This is shown by the thick dashed line in Figure 3(a). Note that these results do not necessarily mean that the same trajectories are realized every time, because these simulations are calculated stochastically.

Figure 3(b) shows the standard deviation of cell volume in one hundred simulations with different random seeds for each permselectivity pattern as a function of time for each permselectivity class. The class of permselectivity is summarized in Table 1 from the view of symmetrical property of permselectivity. The highest values of standard deviation is shown in class 2 at Time $=100,000$ and class 3 follows. The third highest value is in class 5 at Time $=100,000$. Class 1 and class 6 , which have no permselectivity, show relatively lower values than the others.

We define the rate of standard deviation change of cell volume as the rate of diversification, because we can consider that the reproduction rate and the survival rate depend on the cell volume. The rate of diversification in each permselectivity class are shown in Figure 4.

The classes 1 and 6 show the rate of diversification without permselectivity. The classes 2 and 3 exhibit much higher value than the others, but the standard deviations of cell volume often decay halfway through the time in these classes as shown in Figure 3(a) (the case of 'Permselect:0100'). The class 5 has the next higher value. In general, the rates of diversification with permselectivity are 
higher than without permselectivity.

\subsection{Analysis}

We consider in this section how the diversification occurs from the view of correlation between the inner chemical concentration of the cell and the flow of chemicals.

The correlation between the chemical switching and the flow of chemicals is shown in Figure 5(a). It is trivial that the inflow and outflow of chemicals are directly related to the change of cell volume from the definition of the model. And as it is mentioned above that the sets of two chemicals are coupled each other and switching inside cells. The first interesting point is how they are correlated each other. According to Figure 5(a), Class 2 and Class 3 have high correlation between the chemical switching and the flow. This indicates that if the correlation between inner switching and flow is high, the diversification is also high. Note that, however, we do not show "cause and result" but just the correlation between them.

Figure 5(b) shows the ensemble average of inner switching ratio in one hundred simulations with different random seeds in each class. Note that the inner switching ratio is the time average of changing the sign of $\left(x_{1}+x_{3}\right)-\left(x_{2}+x_{4}\right)$ in a cell. We can understand how often the switching averagely occurs in each class from this result. The classes with high deversification rate, i.e., class 2 and class 3, less switch than the other classes. We can consider the mechanism of diversification from these results as follows. A cell has potential of high correlation between the chemical switching and the flow of chemicals in the case that 
the total permeabilities of a set of $\left(\mathrm{X}_{1}, \mathrm{X}_{3}\right)$ and a set of $\left(\mathrm{X}_{2}, \mathrm{X}_{4}\right)$ are different, for example class 2 , class 3 , and class 5 . But the high value of total permeability causes high switching rate, so that the correlation decreases. Therefore, the diversification rates of class 2 and class 3 are relatively high but class 5 is low. If the total permeabilities of the sets are the same, high correlation does not happen. That is why the diversification rates of class 1 , class 4 , and class 6 are low. The situation, of course, will change if the concentration of each chemical in the environment is different.

\section{Conclusion}

We construct a simple autopoietic proto-cell model in which auto-catalytic reactions produce membrane substances, and consider how the membrane permselectivity influences the proto-cell diversification. The simulation results of this model show that the permselectivity of membrane can induce the diversification of the proto-cell volume. This indicates that the reproduction and survival rate of proto-cells can also diversify by the membrane permselectivity if the reproduction and survival rate are assumed to depend on the cell volume even though the internal reaction system does not change. The analyzed date shows two key features which are closely related to the diversification. One of them is the correlation between chemical switching and flow. And the other one is the less number of switching times caused by the low permeability. It is, however, not directly simulated how the diversification is related to the Darwinian evolution yet. We should do the numerical experiment to prove that the variation of 
proto-cells is generated and maintained by the membrane permselectivity and the proto-cells can evolve by the Darwinian evolution. 


\section{References}

[1] P. A. Bachmann, P. L. Luisi, and J. Lang. Autocatalytic self-replicating micelles as models for prebiotic structures. Nature, 357(6373):57-59, 1992.

[2] C. Darwin. On the Origin of Species by Means of Natural Selection or The Preservation of Favored Races in the Struggle for Life. Murray, London, 1859.

[3] T. Gánti. Biogenesis itself. Journal of Theoretical Biology, 187(4):583-593, 1997.

[4] W. Gilbert. Origin of life: The RNA world. Nature, 319(6055):618-618, 1986.

[5] D.T. Gillespie. Exact stochastic simulation of coupled chemical reactions. The Journal of Physical Chemistry, 81(25):2340-2361, 1977.

[6] T. Hashimoto, T. Sato, M. Nakatsuka, et al. Evolutionary constructive approach for studying dynamic complex system. In Giuseppe Petrone and Giuliano Cammarata, editors, Recent Advances in Modelling and Simulation, pages 111-136. I Tech Book, 2008.

[7] K. Kaneko and I. Tsuda. Complex Systems: Chaos and Beyond: A Constructive Approach With Applications in Life Sciences. Springer, 2001.

[8] A. I. Oparin. The Origin of Life. Weidenfeld and Nicolson, London, 1967.

[9] D. Segré, D. Ben-Eli, D. W. Deamer, et al. The lipid world. Origins of Life and Evolution of the Biosphere, 31:119-145, 2001. 
[10] Y. Togashi and K. Kaneko. Alteration of chemical concentrations through discreteness-induced transitions in small autocatalytic systems. Journal of the Physical Society of Japan, 72(1):62-68, 2003.

[11] T. Toyota, K. Takakura, Y. Kageyama, et al. Population Study of Sizes and Components of Self-Reproducing Giant Multilamellar Vesicles. Langmuir, 24(7):3037-3044, 2008.

[12] F. G. Varela, H. R. Maturana, and R. Uribe. Autopoiesis: the organization of living systems, its characterization and a model. Biosystems, 5(4):187196, 1974. 


\section{Tables}

Table 1: Class of permselectivity

\begin{tabular}{cc}
\hline Class & Pattern \\
\hline 1 & 0000 \\
2 & $1000,0100,0010,0001$ \\
3 & 1010,0101 \\
4 & $1100,0110,0011,1001$ \\
5 & $1110,1101,1011,0111$ \\
6 & 1111 \\
\hline
\end{tabular}




\section{Figure Legend}

Figure.1 The image of proto-cell model. $k$ kinds of chemical species react auto-catalytically and some membrane substances are produced from some of chemical species in the auto-catalytic reaction cycle. The numbers in the figure are the chemical species reacting auto-catalytically and ' $\mathrm{M}$ ' means a membrane substance. The thick solid lines are the directions of reaction, the dashed lines indicate the catalyzed reactions, and the circular dotted line means the semipermeable membrane which separates internal and external condition. The thin solid lines across the circular dotted line indicate the permeation of the chemical species.

Figure.2 The time-series behavior of the number of molecules in a cell without permselectivity. (a) Diffusion coefficient $D_{i}=0.001$, (b) $D_{i}=0.0001$ for all $i$.

Figure.3 Examples and standard deviation of cell volume change. (a) Examples of cell volume change as a function of time with permselectivity pattern. (b) Standard deviation of cell volume as a function of time for each class. The 'class' means the type of permselectivity of membern in shown Table 1.

Figure.4 The rate of diversification in each class. The 'class' in this figure is refered to Table 1. 
Figure.5 The frequency of swiching in each class. (a) The correlation coefficients between the concentration inside cell and the flow of chemicals is shown. Specifically speaking, correlation coefficients between $\left(x_{1}+x_{3}\right)-\left(x_{2}+x_{4}\right)$ and (total of inflow - total of outflow) of all chemicals $\left(\mathrm{X}_{1}, \ldots, \mathrm{X}_{4}\right)$ are shown. The correlations of Class 2 and Class 3 are relatively high and the correlations of Class 1 and Class 6, which do not have permselectivity, are relatively low. (b) The average of switching ratio of chemical concentrations $\left(x_{1}+x_{3}\right)$ and $\left(x_{2}+x_{4}\right)$ inside cell. Generally the permeability gets higher, the average also gets higher. 
Figures

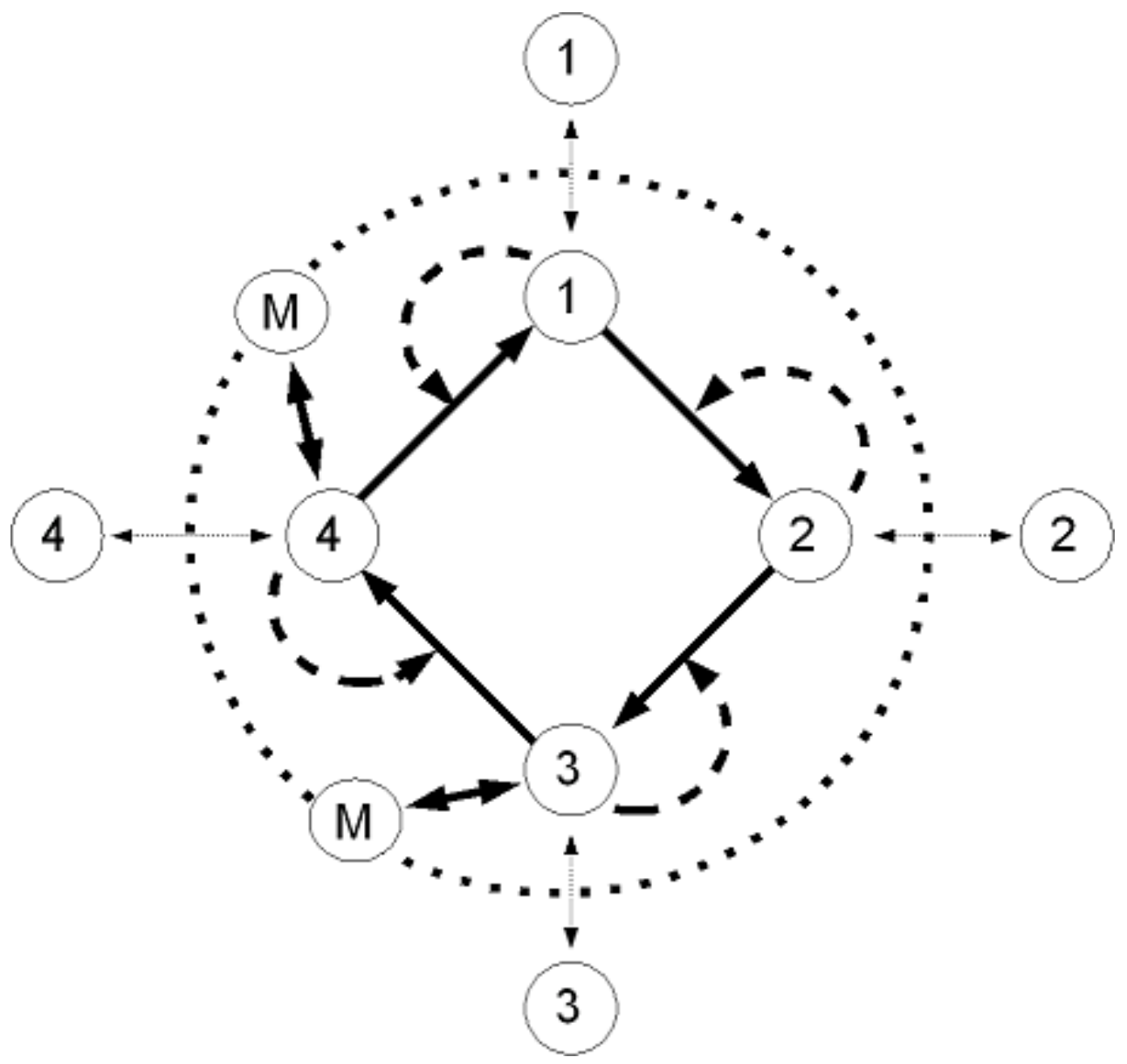

Figure 1: Image of proto-cell model. 


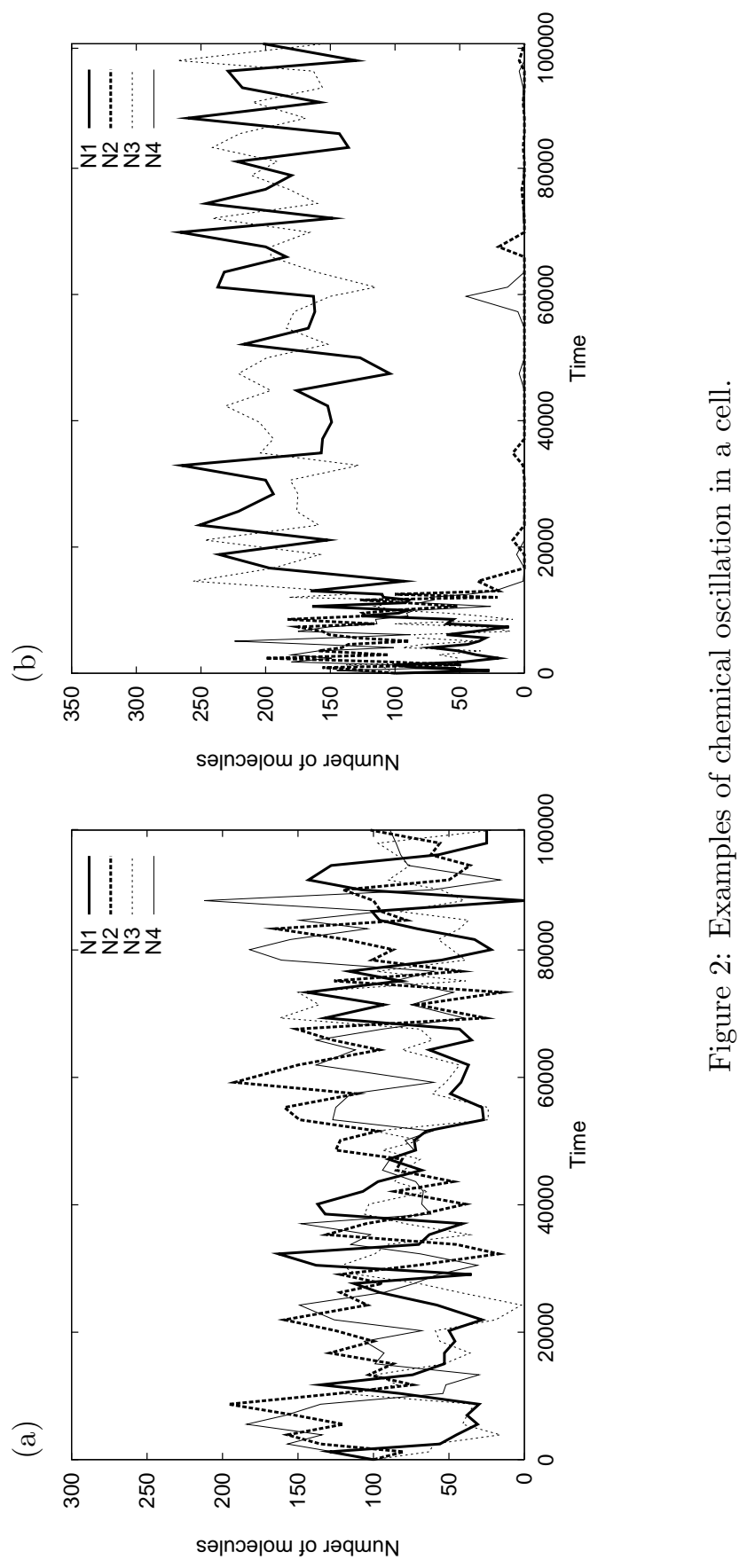




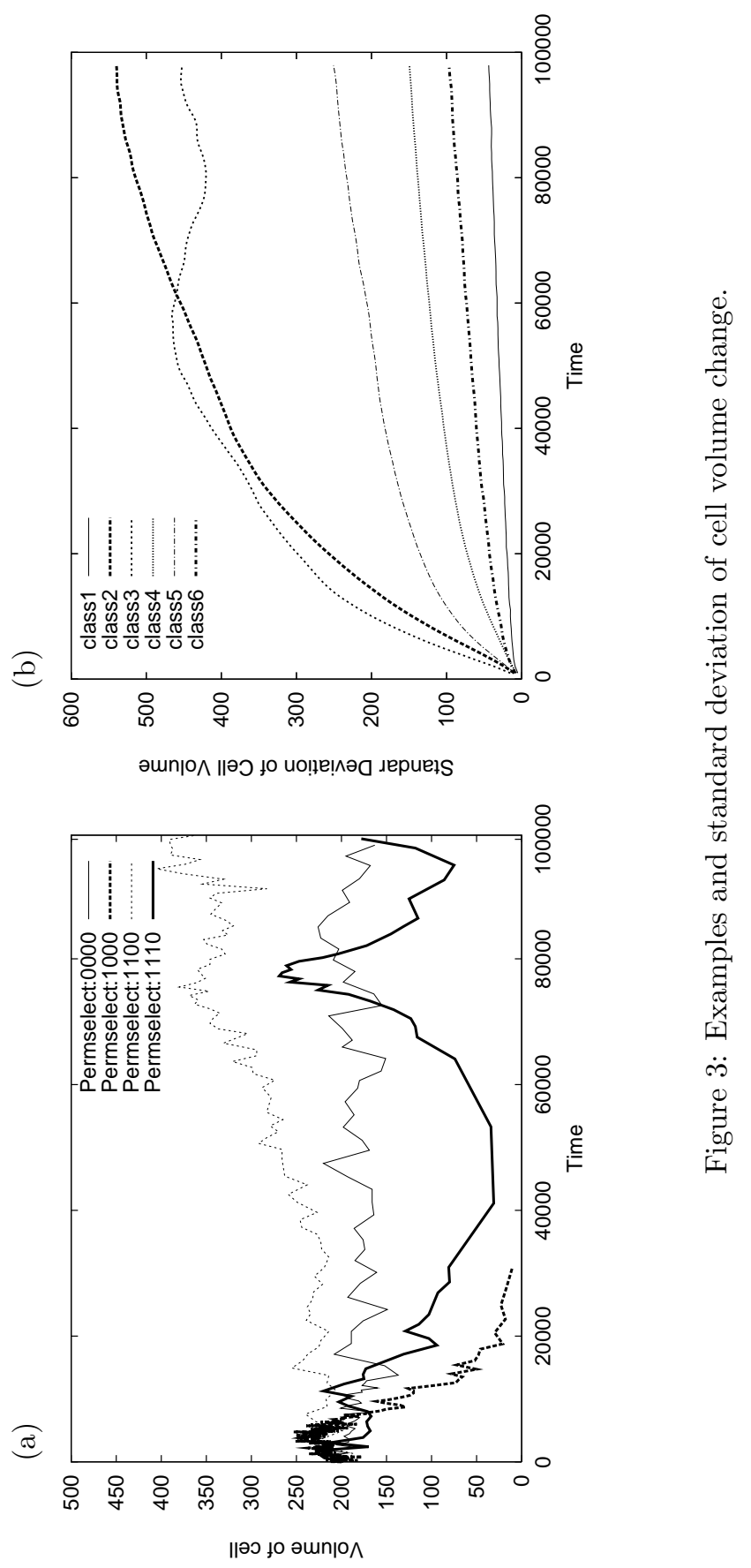


Rate of Diversification

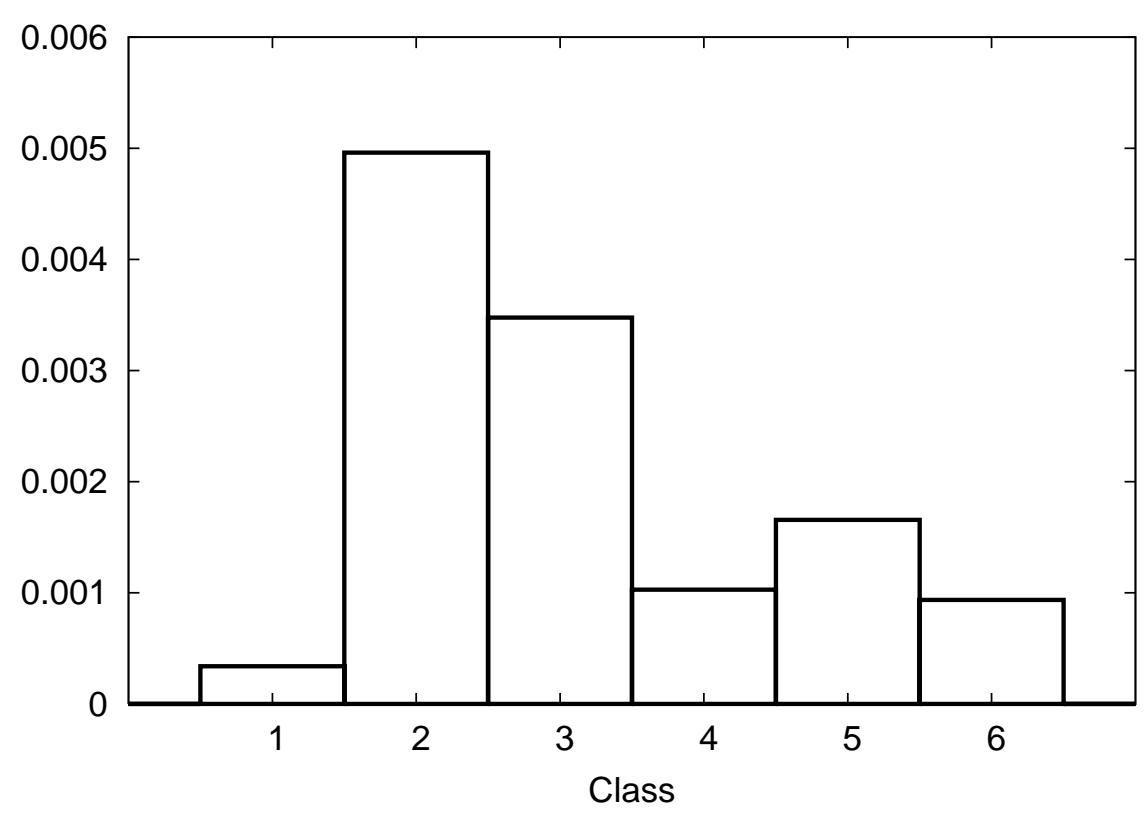

Figure 4: The rate of diversification. 


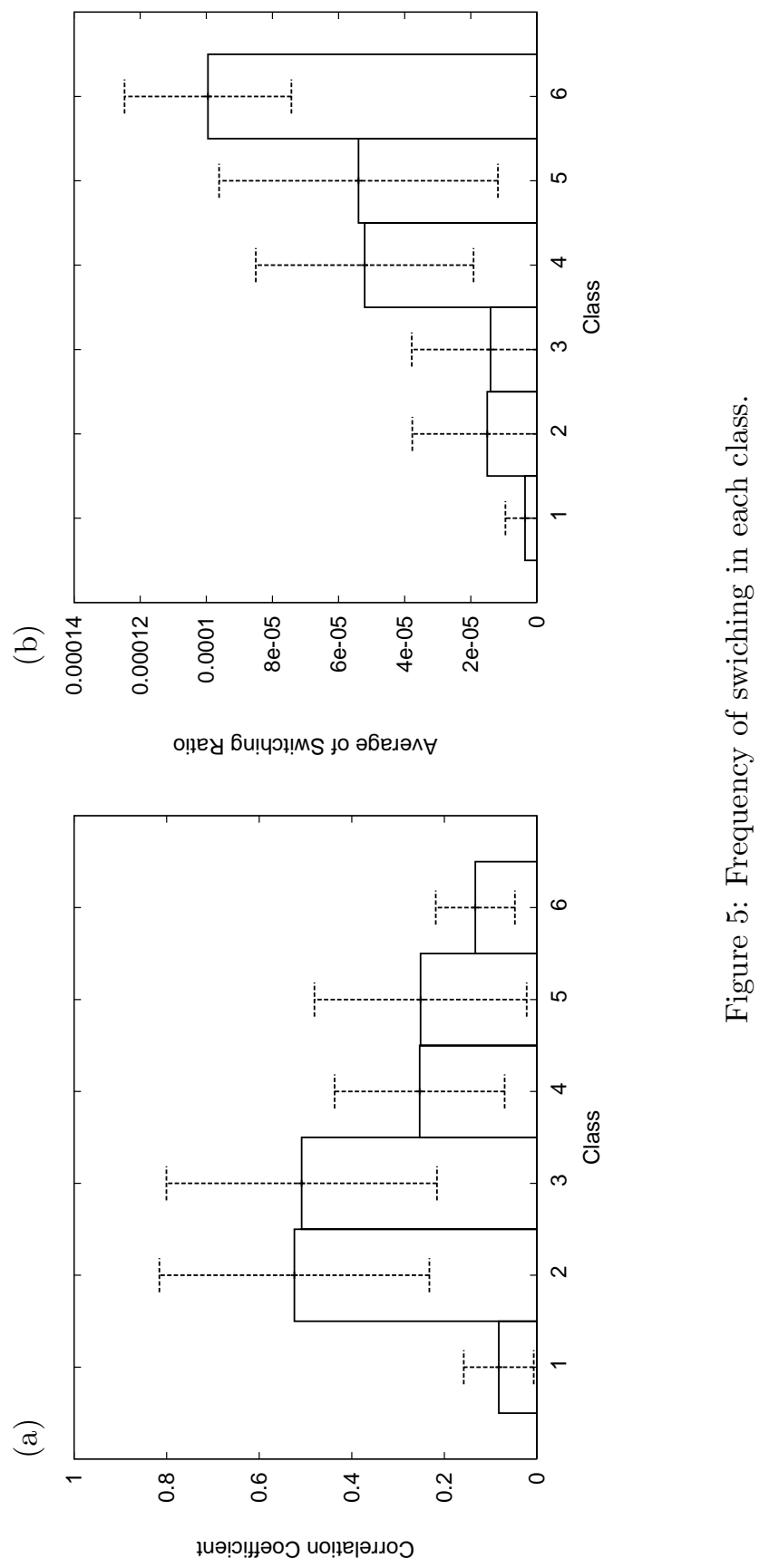

